\title{
Intracerebral Hemorrhage and Subarachnoid Hemorrhage as a Result of Infective Endocarditis: A Case Report
}

\author{
Alviani Februana Waluyani Putri ${ }^{1, *}$, Jagaddhito Probokusumo ${ }^{1}$, Wahyu Widjanarko ${ }^{2}$, Ririn Faujiah ${ }^{2}$ \\ ${ }^{1}$ Medical Doctor at RSUD Jombang, East Java, Indonesia, ${ }^{2}$ Cardiologist at RSUD Jombang, East Java, Indonesia \\ *correspondence: alvianifwp@gmail.com
}

\begin{abstract}
Introduction: Infective endocarditis (IE) may have devastating and life-threatening neurological complications. The incidence of intracranial hemorrhage (e.g. subdural hematoma, subarachnoid hemorrhage $(\mathrm{SAH})$, and intracerebral hemorrhage $(\mathrm{ICH}))$ is rare, occurring in $2.7-7 \%$ of patients with IE.

Case Presentation: A 39 years old male, came to emergency unit with heart failure clinical features, accompanied with fever for 2 weeks, and systolic murmur found in the physical examination. In the second day of admission, the patient experienced a sudden decrease of conciousness, weakness of the right limbs, and slurred speech. CT scan examination showed left parietal lobe $\mathrm{ICH}$ with volume $\pm 20 \mathrm{cc}$, and SAH in left parietal lobe, basal cystern, to pontine cystern. Echocardiography revealed AML flail with severe mitral regurgitation and vegetation in AML. Empirical parenteral antibiotics ceftriaxone and gentamicin were given. In the following day, the conciousness was increasing. Later, the blood culture examination showed growing of Staphylococcus saprophyticus.

Discussion: Intracranial hemorrhage may cause worsening in patient's condition, and require withdrawal in anticoagulant therapy. Cardiac surgery should be delayed.

Conclusion: Despite of its uncommon incidence, physician should be aware of the neurological complication of IE to recognize and do the prompt treatment of the disease.
\end{abstract}

Keywords: infective endocarditis; intracerebral hemorrhage; subarachnoid hemorrhage 